\title{
Investigational Agents for the Treatment of Resistant Yeasts and Molds
}

\author{
Garret T. Seiler $^{1,2}$ (1) $\cdot$ Luis Ostrosky-Zeichner ${ }^{1}$ \\ Accepted: 3 May 2021 / Published online: 28 May 2021 \\ (C) The Author(s), under exclusive licence to Springer Science+Business Media, LLC, part of Springer Nature 2021
}

\begin{abstract}
Purpose of Review This review summarizes the investigational antifungals in clinical development with the potential to address rising drug resistance patterns. The relevant pharmacodynamics, spectrum of activity, preclinical studies, and latest clinical trial data are described.

Recent Findings Agricultural and medicinal antifungal use has been selected for inherently drug-resistant fungi and acquired resistance mechanisms. The rates of fungal infections and immunocompromised populations continue to grow as few new antifungals have hit the market. Several agents with the potential to address the emergence of multidrug-resistant (MDR) molds and yeasts are in clinical development.

Summary Evolved formulations of echinocandins, polyenes, and triazoles offer less toxicity, convenient dosing, and greater potency, potentially expanding these classes' indications. Ibrexafungerp, olorofim, oteseconazole, and fosmanogepix possess novel mechanisms of actions with potent activity against MDR fungi. Successful clinical development is neither easy nor guaranteed; thus, perpetual efforts to discover new antifungals are needed.
\end{abstract}

Keywords Antifungal drugs $\cdot$ Antifungal resistance $\cdot$ Invasive fungal infections $\cdot$ Candida auris $\cdot$ Novel therapies $\cdot$ Review

\section{Introduction}

Only five antifungal classes exist on the market. The polyenes, which destabilize the cell membrane via ergosterol binding, are limited by significant toxicities and intravenous (IV) only formulations for now. Azoles block ergosterol production by inhibiting lanosterol-14 $\alpha$-demethylase (LDM) but often create drug-drug interactions and toxicities by cross-inhibition of mammalian cytochrome P (CYP) enzymes. Echinocandins prevent the biosynthesis of $(1,3) \beta$-d-glucan and are relatively safe but only exist in IV formulations and lack central nervous system (CNS)

This article is part of the Topical Collection on Current Management of Fungal Infections

Garret T. Seiler

gtseiler48@gmail.com

1 Department of Internal Medicine, Division of Infectious Diseases, University of Texas Health Science Center at Houston, McGovern Medical School, Houston, TX, USA

2 Department of Infectious Diseases, Infection Control and Employee Health, The University of Texas MD Anderson Cancer Center, 1515 Holcombe Boulevard, Houston, TX 77030, USA penetration, and their fungicidal spectrum is limited mostly to Candida species. The pyrimidine analog, flucytosine, is indicated only as a combination therapy $[1,2]$. Finally, the allylamines are only used in dermatophyte infections often requiring months of use at the cost of many side effects [2].

Nearly 20 years have passed since the introduction of the newest antifungal class to the market. The incidence of invasive fungal infections (IFI) has since increased along with the immunocompromised population carrying significant mortality rates and costs to healthcare systems $[3,4]$. The estimated annual costs of fungal infections in the USA have reached $\$ 7.2$ billion [5]. With the emergence of multidrug-resistant (MDR) and pan-resistant fungi, there is a critical need for novel antifungals to overcome therapeutic barriers and resistance. In this article, we review fungal resistance patterns and the investigational drugs in clinical development which may rise to meet these challenges.

\section{Epidemiology and Mechanisms of Antifungal Resistance}

The evolution of antifungal resistance is a multifactorial, global phenomenon. Antifungal pesticides in agricultural systems 
and widespread healthcare use have fueled acquired resistance as well as shifted fungal prevalence toward species with inherent antifungal resistance [6]. Climate change and animal reservoirs are also theorized to play an important role, namely, with the global outbreak of $C$. auris [7]. In this section we will describe the known resistance mechanisms of yeast, mold, and dimorphic fungi.

\section{Yeast}

Historically, candidiasis was most often caused by $C$. albicans and was typically sensitive to most azole agents. Widespread fluconazole use has promoted acquired resistance amongst all Candida species and shifted the epidemiology of invasive candidiasis (IC). While C. albicans remains the most common cause of IC, non-albicans species are on the rise (C. glabrata, C. parapsilosis, C. tropicalis, and C. krusei) [8]. C. krusei is intrinsically resistant to fluconazole, and C. glabrata carries a very high rate of resistance. Resistance in Candida species is mediated by the amplification of zinc cluster transcription factors such as UPC2, TAC1, MRR1, or CgPdr1 (particularly in C. glabrata). UPC2 upregulation causes the overexpression of the LDM gene ERG11, while mutations in TAC1, MRR1, and $C g P d r 1$ overexpress drug efflux transporters. Additionally, resistance can occur via altered LDM structure from point mutations in ERG11 [8, 9].

Due to azole resistance, and better clinical outcomes, echinocandins are now the initial drug of choice for IC. Intrinsically elevated minimum inhibitory concentrations (MICs) are present in certain species (C. guilliermondii and C. parapsilosis), yet consistent treatment failures have yet to be demonstrated. On the other hand, mutations in glucan synthase subunits, FKS1 and FKS2, have demonstrated clinically significant echinocandin resistant and MDR isolates [8]. These mutations are more likely to develop with repeated and prolonged drug exposures, especially in those with GI tract biofilm reservoirs [8]. C. auris has burst onto the scene as a major cause of MDR, healthcare-associated candidiasis with mortality rates ranging from 30 to $45 \%$ [10, 11]. Various studies have reported fluconazole resistance 90-93\%, amphotericin $\mathrm{B}(\mathrm{AmB})$ resistance $15-30 \%$, and echinocandin resistance 2-10\% [11].

Polyene resistance amongst yeast is uncommon and typically involves depletion of ergosterol from the cell membrane. It has been seen in Trichosporon species, amongst Candida species (notably $C$. lusitaniae and $C$. auris), and some reports of $C$. neoformans with high MICs [8].

\section{Molds}

The most common mold-related IFI is invasive aspergillosis (IA). Triazoles are the first-line therapy and prophylaxis. While resistance rates remain generally favorable amongst common Aspergillus species (A. fumigatus, A. flavus, A. versicolor, etc.), pan-azole-resistant $A$. fumigatus is increasingly reported in Europe and the USA, and intrinsic triazole resistance is higher amongst cryptic species [12]. The most prevalent azole resistance mechanisms are the overexpression and alteration of the target enzyme gene, Cyp51A, and its promoter region $\left(T R_{46} /\right.$ Tyr121Phe/Thr289Ala; $\mathrm{TR}_{34} /$ Leu98His) [8]. Other mechanisms include biofilm formation, drug efflux, and mutations in transcription factor, HapE [8, 13]. Polyene resistance amongst Aspergillus species typically involves selection for inherently resistant species including A. terreus, A. flavus, and A. nidulans [8].

Scedosporium and Lomentospora species are rare opportunistic molds demonstrating broad and even pan-resistant tendencies with mortality rates breaching $80 \%$. Voriconazole and surgical debridement remain first-line therapies [14]. Similarly, Fusarium species offer a broad spectrum of inherent drug resistances to polyenes, azoles, and echinocandins with variable MICs to triazoles. The mechanisms of this are unclear but theorized to be related to multiple LDM paralogues: CYP51A, B, and C [15].

Mucorales species possess baseline resistance to echinocandins, itraconazole, and voriconazole via alterations in LDM. AmB, posaconazole, and isavuconazole remain the first-line agents, and there is limited data supporting combination therapies [16].

\section{Thermally Dimorphic Fungi}

Endemic fungal diseases such as histoplasmosis, coccidioidomycosis, and paracoccidioidomycosis are either increasing or are underreported $[17,18]$. They generally carry primary resistance to echinocandins, and fluconazole resistance is noted amongst histoplasmosis isolates. Thus, polyenes and azoles remain the preferred agents. While drug resistance remains uncommon, these diseases remain difficult to cure due to the lack of oral polyenes, drug toxicities, and tissue penetration [19].

\section{Investigational Agents}

\section{Agents in Phase III Clinical Trials}

\section{Ibrexafungerp (SCY-078)}

Ibrexafungerp (IBX) is a semi-synthetic derivative of enfumafungin. Similar to echinocandins, IBX inhibits $(1,3) \beta$-d-glucan synthase but via alternative binding sites rendering it unaffected by $F K S$ mutations. It is anticipated to advance the treatment of highly resistant Candida infections; however, its spectrum of activity also includes Cryptococcus species, Aspergillus species, and endemic fungi (Fig. 1) [20]. 
It is soluble at lower $\mathrm{pH}$, enhancing its penetration into acidic environments such as abscesses or vaginal tissue [21]. Although it is a CYP2C8 inhibitor, this effect is unlikely to be clinically significant [22]. Additionally, IBX did not show evidence of reproductive harm in animal models [23].

IBX maintains in vitro activity against fluconazole and echinocandin-resistant Candida species including panresistant $C$. auris $[24 \bullet, 25,26 \bullet]$. It maintained an average $\mathrm{MIC}_{50}$ of $0.5 \mu \mathrm{g} / \mathrm{mL}$ and $\mathrm{MIC}_{90}$ of $1 \mu \mathrm{g} / \mathrm{mL}$ against 100 C. auris isolates and demonstrated increased survival and decreased fungal tissue burden in mouse and guinea pig models of C. auris infections [25]. Amongst 195 C. auris isolates from an outbreak in New York City, 194 were susceptible to IBX with a mean MIC of $0.407 \mu \mathrm{g} / \mathrm{mL}$, including five panresistant isolates [26•].

Advancing to human clinical trials, IBX has been studied in IC, invasive pulmonary aspergillosis (IPA), and vulvovaginal candidiasis (VVC) (Table 1). In a phase II study of IC in nonneutropenic patients, oral IBX demonstrated similar safety and efficacy compared to fluconazole as step-down therapy following echinocandin treatment [27••]. Following a phase Ilb trial demonstrating efficacy and safety IBX $300 \mathrm{mg}$ BID for 1 day versus fluconazole for acute VVC, this dose was carried over to phase III VANISH 303 and 306 trials which established efficacy over placebo with a $50-65 \%$ cure rate determined by test-of-cure cultures [28, 29॰•]. CANDLE is a phase III trial studying IBX in recurrent VVC (NCT04029116).

Oral IBX is currently involved in several active phase III trials. The CARES study is a single-arm, open label study of IBX for invasive $C$. auris infections
(NCT03363841). SCYNERGIA is investigating the combination of IBX and voriconazole versus voriconazole monotherapy in IPA (NCT03672292). Finally, FURI studies the drug for various fungal infections refractory to other therapies (NCT03059992). Positive preliminary data has been released from this study reporting clinical improvement in 17 of 20 patients with esophageal or oropharyngeal candidiasis and intra-abdominal abscesses with the predominant pathogens being C. glabrata and C. krusei $[30 \bullet \cdot$.

\section{Rezafungin (CD101)}

Rezafungin (RZF), developed by Cidara Therapeutics, is the latest echinocandin. Structural modifications have enhanced its chemical stability and solubility granting a long half-life enabling once-weekly IV dosing [31]. This is its greatest novelty as it shares the tissue distribution, favorable safety and drug-drug interaction profiles, and spectrum of activity as other echinocandins (Fig. 1).

Like other echinocandins, RZF demonstrates potent in vitro activity against wild-type and azole-resistant Candida species [32, 33]. FKS1 mutations raise its MIC, although to a lesser degree compared to anidulafungin and micafungin [34]. In mice with invasive $F K S$ mutant C. auris, RZF improved survival and reduced fungal tissue burden compared to micafungin and AmB [35]. Translating RZF to humans with IC has demonstrated safety and efficacy as seen in the phase II STRIVE trial in which the weekly dosing regimen of $400 \mathrm{mg}$ IV for the first week, followed by $200 \mathrm{mg}$ thereafter demonstrated equal, if not slightly better
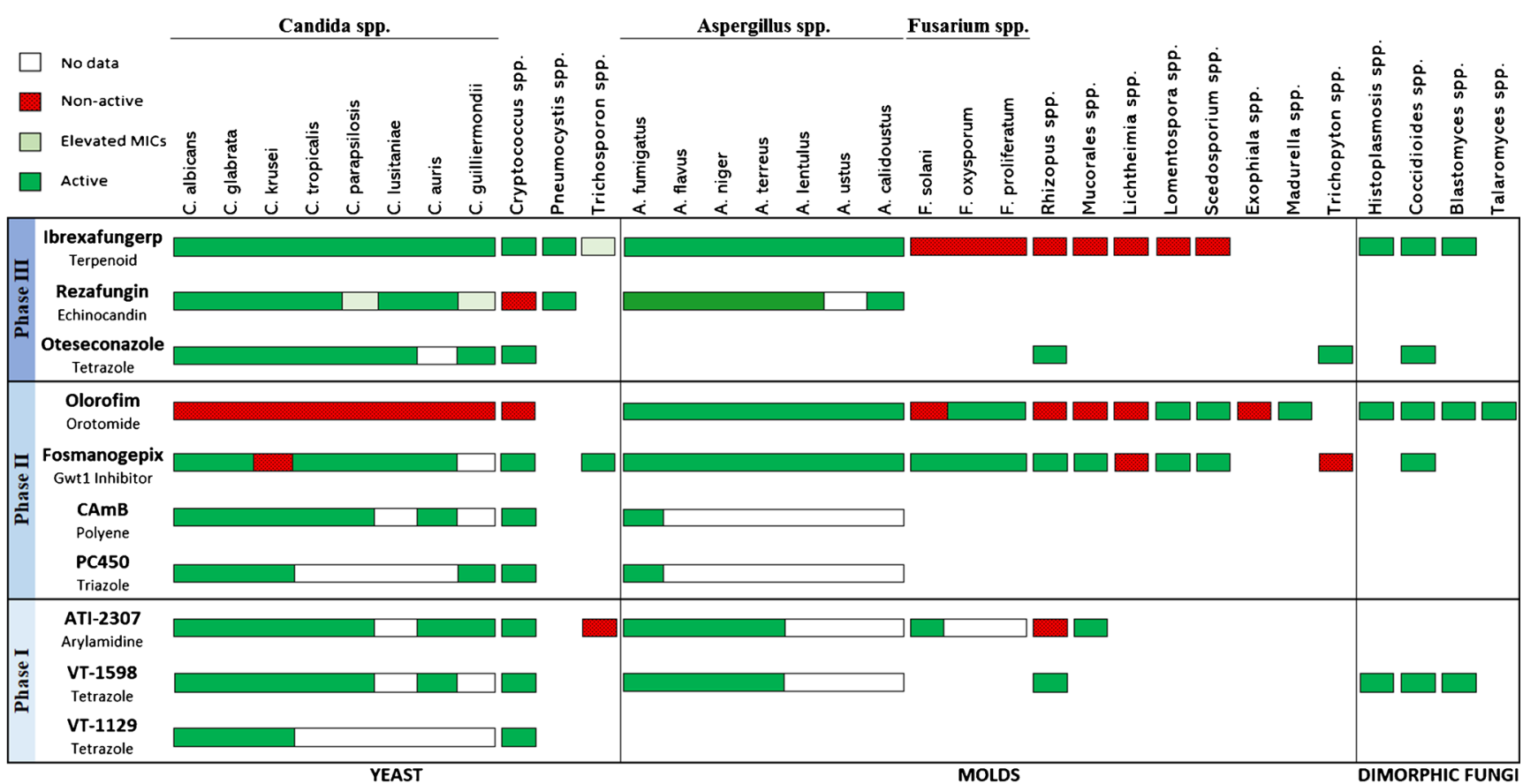

Fig. 1 Spectrum of activity of investigational antifungals organized by their respective phase of clinical development 


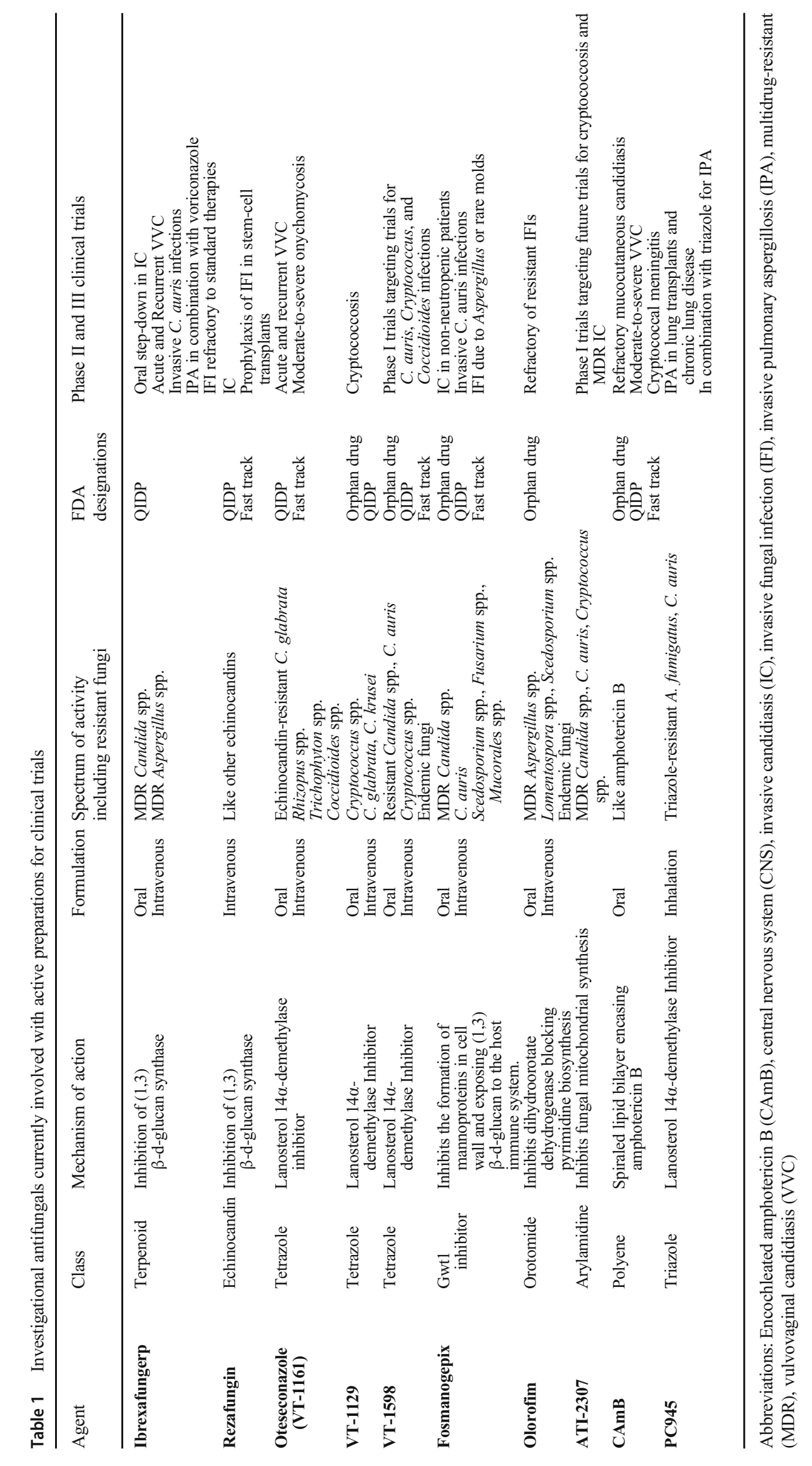


efficacy compared to caspofungin ( $71 \%$ vs $63 \%)$. Species included C. albicans, C. glabrata, C. tropicalis, and C. parapsilosis [36••]. Now RZF has advanced to phase III trials (Table 1). ReSTORE is a multicenter, double blinded, randomized control trial actively recruiting patients with IC to compare weekly IV RZF versus IV caspofungin and stepdown oral fluconazole (NCT03667690).

RZF has been studied in the treatment of other yeasts and molds. Like other echinocandins, it lacks adequate activity against $C$. neoformans [33]. Echinocandins are not used to treat pneumocystis infections due to inactivity against trophic forms [37]. However, RZF has shown activity against Pneumocystis, reducing biofilm mass and inhibiting its production as well as preventing pneumocystis pneumonia (PJP) in immunocompromised mice [38•].

Against Aspergillus species, RZF has not only exhibited in vitro activity against $A$. fumigatus and cryptic species, but also azole-resistant species recovered from the lungs of lung transplant recipients who were taking triazole agents for prophylaxis [39, 40 $]$. Moreover, extended interval dosing of RZF increased survival in a mouse model of azole-resistant disseminated IA [41]. Although these results are encouraging, further phase II and phase III trials are needed to determine its efficacy and safety in human subjects for the prevention and treatment of IA. A phase III trial, ReSPECT, is actively recruiting patient to study RZF's efficacy in the prevention of IFI in allogeneic blood and bone marrow transplant recipients (NCT04368559).

\section{Oteseconazole}

Oteseconazole (VT-1161), developed by Mycovia Pharmaceuticals, belongs to the tetrazole class, a new generation of oral lanosterol $14 \alpha$-demethylase inhibitors which boast higher specificity for fungal CYP51 and therefore theoretically less cross-inhibition of mammalian CYP enzymes leading to fewer drug-drug interactions and adverse effects [42].

Preclinical studies show VT-1161 possess a wide spectrum of activity including Candida species, Rhizopus species, Trichophyton species, and coccidioidomycosis (Fig. 1) [43-46, 47••, 48•]. It exhibited potent in vitro activity against most fluconazole-resistant $C$. albicans and $C$. krusei isolates (mean MIC $\leq 0.15 \mu \mathrm{g} / \mathrm{mL}$ ) as well as echinocandin-resistant C. glabrata $[44,49]$. This effect was demonstrated in a mouse model of VVC which included fluconazole-resistant Candida species [50]. Additionally, VT-1161 has demonstrated in vivo efficacy as prophylaxis against Rhizopus arrhizus infections and coccidioidomycosis treatment $[45,46]$. There is early evidence of possible cross-resistance between triazoles and tetrazoles mediated by target enzyme modification or overexpression, as well as $P D R 1$-mediated drug efflux transporters $[44,49]$.
While VT-1161 is currently in early clinical phases for the treatment of IFIs, it has advanced to phase II and III trials for recurrent VVC and onychomycosis (Table 1). Current treatment options for onychomycosis carry a low success rate. Oral terbinafine or itraconazole offer better efficacy than topical antifungal but at the cost of added toxicities. In a phase II randomized, blinded, placebo-controlled trial of adults with moderate-to-severe onychomycosis, treatment with VT-1161 saw a $42 \%$ cure rate without hepatotoxicity or cardiac toxicity $[47 \cdot \bullet]$. The agent's safety and efficacy were also demonstrated in a phase IIb trial for recurrent VVC [48•]. Three phase III clinical trials of VT-1161 for recurrent VVC are underway (NCT03561701, NCT02267382, NCT03562156).

\section{Agents in Phase II Clinical Trials}

\section{Fosmanogepix}

Fosmanogepix, a prodrug of manogepix, is a first-in-class Gwt1 inhibitor developed by Amplyx Pharmaceuticals. Gwt1 is an inositol acyltransferase which catalyzes posttranslational modification of glycosylphosphatidylinositol (GPI), creating mannoproteins which are anchored into the fungal cell wall, covalently linked to $\beta$-1,3-glucan [51]. These mannoproteins maintain cell wall integrity while facilitating mucosal surface adhesion, biofilm formation, and host invasion. When GPI anchoring is disrupted, $\beta-1,3$-glucan is exposed to the host immune system. It has no activity against the closest mammalian Gwt1 ortholog, GIPW, implying fungal specificity [52]. It has acquired QIDP, orphan drug, and fast track designations for invasive candidiasis, aspergillosis, scedosporiosis, fusariosis, mucormycosis, cryptococcosis, and coccidioidomycosis (Table 1).

Fosmanogepix possesses broad in vitro activity against many genera of fungi: Candida (excluding C. krusei), Trichosporon, Coccidioides, Cryptococcus, Aspergillus (A. fumigatus, A. flavus, A. niger), as well as AmB-resistant strains of Fusarium and Scedosporium (Fig. 1) [51, 53-55]. Amongst Candida species, it maintains activity against echinocandin-resistant FKS mutants; however, elevated MICs were seen with some fluconazole-resistant isolates implicating potential efflux transporter-mediated cross-resistance [56]. Against $C$. auris, fosmanogepix possesses potent in vitro and in vivo activity [54, 57•]. In neutropenic mice with disseminated $C$. auris, this agent increased survivability and lowered fungal tissue burdens compared to echinocandins [54, 58•]. This agent also demonstrates effectiveness against highly resistant mold species. In mouse models of pulmonary scedosporiosis, disseminated fusariosis, and pulmonary mucormycosis, it extended median survival time and decreased fungal burden in lung and brain tissue [59•, 60॰].

Safety, pharmacodynamics, and drug-drug interactions were studied in phase I trials both in healthy individuals and 
patients with acute myelogenous leukemia (NCT02957929, NCT03333005, NCT02956499, and NCT04166669). Fosmanogepix has a higher barrier to acquired resistance, and Gwt1 enzyme mutations do not seem to cause crossresistance amongst other classes [61].

Data from a phase II clinical trial of fosmanogepix for the treatment of invasive candidiasis in non-neutropenic patients reported an $80 \%$ treatment success rate without serious side effects. Additionally, the drug exhibited in vitro activity against all Candida isolates recovered in the study [62••]. Additional phase II trials are currently underway, studying efficacy for IC caused by C. auris (NCT04148287) and IFIs caused by Aspergillus species and other rare molds (NCT04240886).

\section{Olorofim (F901318)}

Olorofim, developed by F2G, is a first-in-class orotomide which affects pyrimidine synthesis via reversible inhibition of dihydroorotate dehydrogenase (DHODH) [63]. This agent has been developed in IV and oral formulations. It possesses time-dependent fungicidal activity and exhibits wide tissue distribution (including the CNS), with enterohepatic recirculation. Many molds and endemic fungi are within its spectrum of activity, but it lacks reliable activity against yeast (Fig. 1) [64]. It has been granted QIDP and orphan drug status for the treatment of coccidioidomycosis, invasive Aspergillus, Scedosporium, and Lomentospora species infections (Table 1).

Olorofim shows great promise for the treatment of resistant mold infections. It possesses activity against highly resistant and cryptic Aspergillus species including A. lentulus, A. fumigatiaffinis, and A. calidoustus [65•]. In vitro activity has been demonstrated against triazole-resistant $A$. fumigatus (MIC $\leq 0.008-0.03 \mathrm{mg} / \mathrm{L}$ ), L. prolificans (MIC 0.03-0.5mg/ L), Scedosporium species (MIC 0.03-0.5mg/L), Rasamsonia argillacea $(\mathrm{MIC} \leq 0.008-0.03 \mathrm{mg} / \mathrm{L})$, and certain Fusarium species (F. oxysporum complex but not $F$. solani) [66•, 67]. Against Lomentospora prolificans biofilms, this agent demonstrates greater penetration than $\mathrm{AmB}$ and micafungin [68•]. In vivo studies of olorofim in neutropenic mouse models of IPA (wild-type and triazole-resistant mutants), sinopulmonary A. flavus, and chronic granulomatous disease-related IPA ( included resistant cryptic species $A$. nidulans and A. tanneri) demonstrated efficacy with reductions in galactomannan and mortality which were predicted by $\mathrm{C}_{\min } / \mathrm{MIC}$ values [68, 69 , 70]. It is important to note this drug lacks activity against Mucorales species or Exophiala dermatitidis due to phylogenetically different DHODH target enzymes [63, 65].

Regarding endemic fungal diseases, olorofim exhibits low MICs for Histoplasma capsulatum, Blastomyces species, Coccidioides species, and Talaromyces marneffei [71]. In a murine model, olorofim demonstrated increased survival of mice with CNS coccidioidomycosis, a clinical entity notoriously difficult to completely cure $[72,73]$.

Nine phase I clinical trials of olorofim have been completed. Tolerability, safety, and pharmacodynamics have been assessed for single and multiple ascending doses of oral and IV formulations (NCT02142153, NCT0239448, NCT02342574, NCT02737371, NCT02808741). Olorofim exerts a mild inhibitory effect on CYP3A4 (NCT02680808, NCT04171739).

A phase IIb trial is currently underway enrolling patients with invasive fungal infections which are refractory or resistant to standard therapy (NCT03583164; FORMULA-OLS).

\section{Encochleated Amphotericin B}

Encochleated amphotericin B (CAmB), developed by Matinas Biopharma, is a novel oral polyene formulation in which AmB and calcium are encased within a cochleate (spiraled, negatively charged lipid bilayer). The cochleate stabilizes, protects, and delivers the drug into reticuloendothelial cells. Thus, low levels of active drug circulate in plasma mitigating the adverse effects commonly seen in $\mathrm{AmB}$ toxicities. As the compound is phagocytosed, calcium gradients facilitate the release of AmB into phagocytes and the extracellular space. This agent demonstrates broad tissue penetration including the brain, kidney, lung, and bone with a spectrum of activity akin to AmB (Fig. 1) [74].

The safety and efficacy of $\mathrm{CAmB}$ have been demonstrated in murine models of IA, IC, and cryptococcal meningitis [75-77]. One hundred percent of mice with IC treated with $\mathrm{CAmB}$ at doses ranging from 0.5 to $5 \mathrm{mg} / \mathrm{kg} /$ day survived with a dose-dependent decrease in kidney and lung fungal tissue burden [76]. CAmB was found to be equally efficacious as AmB with superiority over oral fluconazole in cryptococcal meningoencephalitis [77].

In a recent phase I study, HIV patients with prior history of cryptococcosis were administered different dosing regimens of CAmB: single administrations of ascending daily doses $(1.0 \mathrm{~g}$, $1.5 \mathrm{~g}$, or $2.0 \mathrm{~g}$ ) split into 4-6 divided doses and recurrent doses of $1.5 \mathrm{~g}$ daily split into 4-6 divided doses over 7 days. Excellent tolerability (98-100\%) and improved safety compared to AmB were demonstrated. No nephrotoxicity was reported [78•].

Preliminary results of a phase IIa trial investigating CAmB in refractory mucocutaneous candidiasis demonstrated tolerability and improvement in symptoms without drug-related toxicities outside of nausea or dizziness [79]. Another phase II trial compared safety and efficacy of single doses of CAmB $200 \mathrm{mg}$, CAmB $400 \mathrm{mg}$, and fluconazole $150 \mathrm{mg}$ for moderate-tosevere VVC. There were no serious adverse events reported, but clinical cure rates favored fluconazole over CAmB $200 \mathrm{mg}$ and CAmB $400 \mathrm{mg}$ (75\%, 52\%, and 54\%, respectively) [80]. An additional phase II clinical trial studying CAmB in cryptococcal meningitis is underway (NCT04031833). 


\section{PC945}

PC945 is the first topical, inhaled triazole. Developed by Pulmocide, this drug is designed primarily for the treatment of IPA, boasting prolonged lung tissue retention without significant systemic absorption. Its antifungal spectrum is analogous to systemic triazoles but with significantly greater potency maintaining activity against posaconazole and pan-azoleresistant A. fumigatus, as well as C. auris (Fig. 1) [81, 82]. Synergy with systemic triazoles was demonstrated in murine IPA models [83]. Following successful phase I clinical trials, phase II trials for IPA in patients with cystic fibrosis, chronic lung disease, and lung transplants have been terminated early due to the COVID-19 pandemic. A phase III trial with PC945 in combination with systemic antifungals for patients with IPA and no other treatment options planned (Table 1) [84].

\section{Antifungals in Phase I and Preclinical Development}

\section{ATI-2307}

ATI-2307 was originally developed by the FUJIFILM Toyama Chemical Co. under the name T-2307 until November 2019 when Appili Therapeutics Inc. acquired the development rights and renamed it. It is a first-in-class arylamidine with a novel antifungal mechanism: the disruption of yeast cellular respiration via inhibition of mitochondrial respiratory chain complexes III and IV. This is speculated to be a fungal-specific effect [85]. Preclinical studies have demonstrated potent, fungicidal in vitro and in vivo activity against azole and echinocandin-resistant $C$. glabrata, C. auris, C. neoformans, C. gattii, and A. fumigatus (Fig. 1) $[86-90,91 \bullet]$. Its application to human subjects has been targeted at cryptococcosis and invasive MDR candidiasis. Phase I clinical trials to determine safety, efficacy, and optimal human doses are currently underway with preliminary reports from a trial with cryptococcal meningitis indicating superior potency and acceptable safety compared to standard-of-care therapies (Table 1). Phase II clinical trials are anticipated to begin in 2022 [91•].

\section{Quilseconazole (VT-1129)}

VT-1129 is an oral tetrazole being studied for the treatment of cryptococcal meningitis. The FDA has granted it orphan drug status and QIDP designation for this purpose. Preclinical studies have demonstrated robust in vitro and in vivo efficacy against $C$. neoformans and $C$. gattii, notably maintaining activity (mean MIC $0.027 \mu \mathrm{g} / \mathrm{mL}$ ) against fluconazole-resistant or dose-dependent $C$. neoformans isolates (Fig. 1) [92-94]. In mice treated with doses $\geq 3 \mathrm{mg} / \mathrm{kg}$ daily, there was virtually no evidence of fungal burden in CNS tissues at the day of medication cessation or 20 days later, a finding not seen in fluconazole-treated mice [94]. Additionally, in vitro studies denote activity against Candida species isolated from patients with chronic mucocutaneous candidiasis as well as C. glabrata and C. krusei isolates resistant to azoles and echinocandins $[44,95]$.

\section{VT-1598}

VT-1598, another oral tetrazole, boasts a wide antifungal spectrum including yeast, molds, and dimorphic fungi. It has been granted FDA fast track status for the treatment of coccidioidomycosis (Table 1). It is currently in phase I trials for potential use in C. auris infections, cryptococcosis, and coccidioidomycosis (NCT04208321).

In preclinical studies, VT1598 effectively inhibited Candida species (C. glabrata, C. parapsilosis, C. krusei, C. auris, and azole-resistant C. albicans), Cryptococcus species, Aspergillus species (A. fumigatus, A. flavus, A. niger, and A. terreus), Rhizopus arrhizus (except $R$. arrhizus var. delemar strains), Coccidioides species, $B$. dermatitis, and H. capsulatum (Fig. 1) [49, 96-98]. Like VT-1161, it is impacted by UPC2- and PDR1-mediated resistance mechanisms seen in triazole cross-resistant $C$. glabrata [97]. Efficacy and safety were seen in mouse models studying VT-1598 for invasive $C$. auris infections, cryptococcal meningitis, and CNS coccidioidomycosis [98-100].

\section{Miscellaneous Agents Not in Active Clinical Trials}

\section{Nikkomycin Z}

Nikkomycin $\mathrm{Z}$ is a first-in-class chitin-synthase inhibitor developed by Valley Fever Solutions as a targeted approach against endemic fungi, including Coccidioides species [101]. Additionally, it found a synergistic role in treating resistant Candida species. Preclinical studies showed efficacy in canine pulmonary coccidioidomycosis and murine echinocandinresistant $F K S$ mutant invasive candidiasis [102, 103]. While the initial phase I trial exhibited adequate safety, additional phase I and phase II trials were unable to recruit patients and lacked sufficient funding to continue [104]. With renewed interest and support, this agent could recrudesce into clinical development again.

\section{MGCD290}

MGCD290 is an inhibitor of fungal histone deacetylase. It showed early promise in preclinical trials as a synergistic agent restoring triazole susceptibility in Candida, Fusarium, and Zygomycetes species as well as echinocandin susceptibility in resistant Candida species [105, 106]. However, a phase II study of MGCD290 plus fluconazole in VVC did not show superiority compared to fluconazole alone (NCT01497223). Presently, there are no active clinical trials. 


\section{Conclusions}

This arsenal of agents offers hope for the future. The rise of MDR and pan-resistant Candida species has been met with advanced stage investigational agents such as IBX, VT-1161, and RZF. IBX introduces a novel mechanism, potent broad spectrum of activity and a safe oral formulation, while VT-1161 is poised to treat recurrent VVC and onychomycosis with potential to garner other indications as clinical development progress. RZF offers convenient, once-weekly dosing for echinocandin for the treatment or prophylaxis in immunocompromised patients.

Perhaps the brightest future for the future of invasive mold infections lie in stage II and I agents fosmanogepix and olorofim, both first agents in their respective classes. Fosmanogepix has displayed efficacy in IC and is currently involved in trials for invasive C. auris and mold infections. Fosmanogepix and olorofim also possess activity against notoriously MDR panresistant mold genera, Lomentospora and Scedosporium. Currently, infections by these organisms have few, if any, reliable therapeutic options.

The reformulation of existing classes into new delivery systems will change the treatment of IFIs. CAmB offers safe oral administration of AmB, and PC945 is a potent inhaled topical triazole with minimal systemic absorption.

From conceptualization to proof of efficacy in humans, antifungal development is an expensive and protracted challenge. Many promising antifungals never reach the market due to poor recruitment, lack of funding, or trial failures. While these agents are a great start, with the rising immunocompromised population and perpetual evolution of antifungal resistance, it is essential that continual efforts are made toward the discovery of new therapies.

\section{Declaration}

Conflict of interest GTS does not have any conflict of interest to report. LO has received speaking, consulting, and/or research funds from Pfizer, Astellas, F2G, Amplyx, Cidara, Scynexis, and Gilead.

Human \& Animal Rights and Informed Consent This article does not contain any studies with human or animal subjects performed by any of the authors.

\section{References}

Papers of particular interest, published recently, have been highlighted as:

- Of importance

•- Of major importance

1. Mourad A, Perfect J. Tolerability profile of the current antifungal armoury. Journal of Antimicrobial Chemotherapy. 2018;73:i2632.
2. Gintjee T, Donnelley M, Thompson G. Aspiring antifungals: review of current antifungal pipeline developments. Journal of Fungi. 2020;6:28.

3. Vallabhaneni S, Mody R, Walker T, Chiller T. The global burden of fungal diseases. Infectious Disease Clinics of North America. 2016;30:1-11.

4. Lum L, Lee A, Vu M, Strasser S, Davis R. Epidemiology and risk factors for invasive fungal disease in liver transplant recipients in a tertiary transplant center. Transpl Inf Dis. 2020:e13361.

5. Benedict K, Jackson BR, Chiller T, Beer KD. Estimation of direct healthcare costs of fungal diseases in the United States. Clin Inf Dis. 2019;68(11):1791-7. https://doi.org/10.1093/cid/ciy776.

6. Verweij PE, Snelders E, Kema GH, Mellado E, Melchers WJ. Azole resistance in Aspergillus fumigatus: a side-effect of environmental fungicide use? The Lancet Infectious Diseases. 2009;9:789-95.

7. Casadevall A, Kontoyiannis DP, Robert V. On the emergence of Candida auris: climate change, azoles, swamps and birds. mBio. 2019;10:e01397-19.

8. Perlin DS, Rautemaa-Richardson R, Alastruey-Izquierdo A. The global problem of antifungal resistance: prevalence, mechanisms, and management. The Lancet Infectious Diseases. 2017;17:e383-92.

9. Whaley SG, Berkow EL, Rybak JM, Nishimoto AT, Barker KS, Rogers PD. Azole antifungal resistance in Candida albicans and emerging non-albicans Candida species. Frontiers in Microbiology. 2017;7. https://doi.org/10.3389/fmicb.2016.02173.

10. Adams E, Quinn M, Tsay S, Poirot E, Chaturvedi S, Southwick K, et al. Candida auris in healthcare facilities, New York, USA, 2013-2017. Emerging Infectious Diseases. 2018;24(10):181624. https://doi.org/10.3201/eid2410.180649.

11. Forsberg K, Woodworth K, Walters M, Berkow EL, Jackson B, Chiller T, et al. Candida Auris : The recent emergence of a multidrug-resistant fungal pathogen. Medical Mycology. 2019;57(1):1-12. https://doi.org/10.1093/mmy/myy054.

12. Walker TA, Lockhart SR, Beekmann SE, Polgreen PM, Santibanez S, Mody RK, et al. Recognition of azole-resistant aspergillosis by physicians specializing in infectious diseases, United States. Emerging Infectious Diseases. 2018;24.

13. Hortschansky P, Misslinger M, Mörl J, Gsaller F, Bromley M, Brakhage A, et al. Structural basis of HapE P88L-linked antifungal triazole resistance in Aspergillus fumigatus. Life Science Alliance. 2020;3:e202000729. https://doi.org/10.26508/1sa. 202000729.

14. Ramirez-Garcia A, Pellon A, Rementeria A, Buldain I, BarretoBergter E, Rollin-Pinheiro R, et al. Scedosporium and Lomentospora: an updated overview of underrated opportunists. Medical Mycology. 2018;56(1):102-25.

15. Al-Hatmi AMS, de Hoog GS, Meis JF. Multiresistant Fusarium pathogens on plants and humans: solutions in the antifungal pipeline? Infect Drug Resist. 2019;12:3727-37. https://doi.org/10. 2147/IDR.S180912.

16. Schwarz P, Cornely OA, Dannaoui E. Antifungal combinations in Mucorales: a microbiological perspective. Mycoses. 2019;3:74660.

17. Tirado-Sánchez A, González GM, Bonifaz A. Endemic mycoses: epidemiology and diagnostic strategies. Expert Review of Antiinfective Therapy. 2020:1-13.

18. CDC. Increase in reported coccidioidomycosis - United States, 1998-2011. MMWR. 2013;62(12):217-21.

19. Parente-Rocha, Alves J, et al. Antifungal resistance, metabolic routes as drug targets, and new antifungal agents: an overview about endemic dimorphic fFungi. Mediators of Inflammation. 2017:1-16. https://doi.org/10.1155/2017/9870679.

20. Davis MR, Donnelley MA, Thompson GR. Ibrexafungerp: a novel oral glucan synthase inhibitor. Medical Mycology. 2020;58(8): 579-92. 
21. Wring SA, Randolph R, Park S, et al. Preclinical pharmacokinetics and pharmacodynamic target of SCY-078, a first-in class orally active antifungal glucan synthesis inhibitor, in murine models of disseminated candidiasis. Antimicrob Agents Chemother. 2017;61:e02068-16.

22. Wring S, Murphy G, Atiee G, Corr C, Hyman M, Willett M, et al. Lack of impact by SCY-078, a first-in-class oral fungicidal glucan synthase inhibitor, on the pharmacokinetics of rosiglitazone, a substrate for CYP450 2C8, supports the low risk for clinically relevant metabolic drug-drug interactions. The Journal of Clinical Pharmacology. 2018;58:1305-13.

23. Carruthers C, Barat S, Angulo D, Thomas P, Lewis E, Sobel R. Ibrexafungerp, a novel oral antifungal, demonstrates no reproductive or developmental harm in preclinical models. Obstetrics \& Gynecology. 2019;133:114S.

24. Nunnally NS, Etienne KA, Angulo D, et al. In vitro activity of ibrexafungerp, a novel glucan synthase inhibitor against Candida glabrata isolates with FKS mutations. Antimicrob Agents Chemother. 2019;63:11. https://doi.org/10.1128/AAC.01692-19 Ibrexafungerp has activity against echinocandin-resistant $\boldsymbol{C}$. glabrata.

25. Barat S, Borroto-Esoda K, Angulo D. SCY-078 Demonstrates significant tissue penetration in rats and mice following oral or IV administration. Open Forum Infectious Diseases. 2017;4: S471-1.

26. Zhu YYC, Barat SA, Borroto-Esoda K, Angulo D, Chaturvedi S, Chaturvedi V. Pan-resistant Candida auris isolates from the outbreak in New York are susceptible to ibrexafungerp (a glucan synthase inhibitor). Int J Antimicrob Agents. 2020;55(4): 105922. https://doi.org/10.1016/j.ijantimicag Ibrexafungerp maintained against pan-resistant $C$. auris isolated from human patients in New York.

27.• Spec A, Pullman J, Thompson GR III, Powderly WG, Tobin EH, Vazquez J, et al. MSG-10: a phase 2 study of oral ibrexafungerp (SCY-078) following initial echinocandin therapy in nonneutropenic patients with invasive candidiasis. J of Antimicrob Chemother. 2019;74(10):3056-62 Ibrexafungerp showed efficacy in phase II study for invasive candidiasis.

28. Cadet APA, Rebecca BA, Tufa MMD, Angulo DMD, Nyirjesy PMD. A Phase $2 b$, Dose-finding study evaluating oral ibrexafungerp vs fluconazole in vulvovaginal candidiasis (DOVE). Obstetrics \& Gynecology. 2019;133:113S-4S. https:// doi.org/10.1097/01.AOG.0000558840.33387.ee.

29.• Trialsitenews.com (2020) Scynexis announces positive results from phase 3 VANISH-306 study of oral ibrexafungerp to treat vulvovaginal candidiasis. https://www.trialsitenews.com/ scynexis-reports-positive-results-from-phase-3-vanish-306-trialof-oral-ibrexafungerp-for-vaginal-yeast-infection. Accessed 11 Aug 2020. Oral ibrexafungerp showed efficacy in phase III study for VVC.

30.• Synexis.com (2019) SCYNEXIS announces positive interim results from Phase 3 open-label FURI study. https://www.scynexis. com/news-media/press-releases/detail/169/scynexis-announcespositive-interim-results-from-phase-3. Accessed 11 Aug 2020. Early data from phase III study show ibrexafungerp is efficacious in invasive fungal infections resistant or refractory to other antifungals.

31. Krishnan BR, James KD, Polowy K, Bryant BJ, Vaidya A, Smith $\mathrm{S}$, et al. CD101, a novel echinocandin with exceptional stability properties and enhanced aqueous solubility. J Antibiot. 2017;70: $130-5$.

32. Tóth Z, Forgács L, Locke JB, Kardos G, Nagy F, Kovács R, et al. In vitro activity of rezafungin against common and rare Candida species and Saccharomyces cerevisiae. J Antimicrob Chemother. 2019;74:3505-10.
33. Pfaller MA, Carvalhaes CG, Messer SA, Rhomberg PR, Castanheira M. Activity of a long-acting echinocandin rezafungin and comparator antifungal agents tested against contemporary invasive fungal isolates: (SENTRY Program, 2016 to 2018). Antimicrob Agents Chemother. 2020;64.

34. Helleberg M, Jørgensen KM, Hare RK, Datcu R, Chowdhary A, Arendrup MC. Rezafungin In vitro activity against contemporary Nordic clinical Candida isolates and Candida auris determined by the EUCAST reference method. Antimicrob Agents Chemother. 2020;64.

35. Hager CL, Larkin EL, Long LA, Ghannoum MA. Evaluation of the efficacy of rezafungin, a novel echinocandin, in the treatment of disseminated Candida auris infection using an immunocompromised mouse model. J Antimicrob Chemother. 2018;73:2085-8.

36.• Thompson GR, Vazquez J, Soriano A, Skoutelis A, OstroskyZeichner L, Mena K, et al. Rezafungin clinical safety and efficacy in patients with candidemia and/or invasive candidiasis in the randomized, double-blind, multicenter, phase 2 STRIVE study. Open Forum Infect Dis 2018;5. Rezafungin is effective and safe for patients with candidemia or invasive candidiasis.

37. Cushion MT, Collins MS. Susceptibility of pneumocystis to echinocandins in suspension and biofilm cultures. Antimicrob Agents Chemother. 2011;55:4513-8.

38. Cushion M, Ashbaugh A. Rezafungin prophylactic efficacy in a mouse model of pneumocystis pneumonia. Biol Blood Marrow Tr. 2019;25 Preclinical data suggesting a potential role of rezafungin for pneumocystis pneumonia prophylaxis, offering a potential single agent prophylaxis regimen for certain stem cell transplant recipients or other immunocompromised patients.

39. Wiederhold NP, Locke JB, Daruwala P, Bartizal K. Rezafungin (CD101) demonstrates potent in vitro activity against Aspergillus, including azole-resistant Aspergillus fumigatus isolates and cryptic species. J Antimicrob Chemother. 2018;73:3063-7.

40. Driscoll E, Clancy CJ, Nguyen M-H. 738. Potent in vitro activity of rezafungin (RZF) against Aspergillus clinical isolates recovered from lung transplant patients who have received $\geq 3$ months of triazole prophylaxis. Open Forum Infect Dis. 2019;6 Rezafungin may have potential as salvage therapy for invasive mold infections breaking through triazole prophylaxis.

41. Wiederhold NP, Najvar LK, Jaramillo R, Olivo M, Wickes BL, Catano $\mathrm{G}$, et al. Extended-interval dosing of rezafungin against azole-resistant Aspergillus fumigatus. Antimicrob Agents Chemother. 2019;63.

42. Warrilow AG, et al. The clinical candidate VT-1161 is a highly potent inhibitor of Candida albicans CYP51 but fails to bind the human enzyme. Antimicrob Agents Chemother. 2014;58(12): 7121-7. https://doi.org/10.1128/aac.03707-14.

43. Warrilow AG, Parker JE, Price CL, et al. The tetrazole VT-1161 is a potent inhibitor of Trichophyton rubrum through its inhibition of T. rubrum CYP51. Antimicrob Agents Chemother. 2017;61: e00333-17.

44. Schell WA, Jones AM, Garvey EP, et al. Fungal CYP51 inhibitors VT-1161 and VT-1129 exhibit strong in vitro activity against Candida glabrata and C. krusei isolates clinically resistant to azole and echinocandin antifungal compounds. Antimicrob Agents Chemother. 2017;61:e01817-6.

45. Gebremariam T, Wiederhold NP, Fothergill AW, Garvey EP, Hoekstra WJ, Schotzinger RJ, et al. VT-1161 protects immunosuppressed mice from Rhizopus arrhizus var. arrhizus infection. Antimicrob Agents Chemother. 2015;59:7815-7.

46. Shubitz LF, Roy ME, Trinh HT, Hoekstra WJ, Schotzinger RJ, Garvey EP. Efficacy of the investigational antifungal VT-1161 in treating naturally occurring coccidioidomycosis in dogs. Antimicrob Agents Chemother. 2017;61:e00111-7. https://doi. org/10.1128/AAC.00111-17. 
47. Elewski B, Brand S, Degenhardt T, Curelop S, Pollak R, Schotzinger R, et al. A phase II, randomized double-blinded, placebo controlled, dose-ranging study to evaluate the efficacy and safety of VT-1161 oral tablets in the treatment of patients with distal and lateral subungual onychomycosis of the toenail. Br J Dermatol. 2020. https://doi.org/10.1111/bjd.19224 Phase II data showing efficacy for moderate to severe onychomycosis. Current antifungals for onychomycosis have low success rate, systemic toxicities and are primarily indicated for mild-moderate disease.

48. Brand SR, Degenhart TP, Person KL, et al. Oral VT-1161 is highly effective and safe in patients with recurrent vulvovaginal candidiasis - results of REVIVE, a multicenter phase $2 b$ study. ASM Microbe. New Orleans: Viamet Pharmaceuticals. 2017; Phase II data supporting its use for recurrent vaginal candidiasis.

49. Nishimoto AT, Wiederhold NP, Flowers SA, Zhang Q, Kelly SL, Morschhäuser J, et al. In vitro activities of the novel investigational tetrazoles vt-1161 and vt-1598 compared to the triazole antifungals against azole-resistant strains and clinical isolates of Candida albicans. Antimicrob Agents Chemother. 2019;63:1-11.

50. Garvey EP, Hoekstra WJ, Schotzinger RJ, Sobel JD, Lilly EA, Fidel PL Jr. Efficacy of the clinical agent VT-1161 against fluconazole-sensitive and -resistant Candida albicans in a murine model of vaginal candidiasis. Antimicrob Agents Chemother. 2015;59:5567-73.

51. Miyazaki M, Horii T, Hata K, Watanabe NA, Nakamoto K, Tanaka K, et al. In vitro activity of E1210, a novel antifungal, against clinically important yeasts and molds. Antimicrob Agents Chemother. 2011;55:4652-8.

52. Rivero-Menendez O, Cuenca-Estrella M, Alastruey-Izquierdo A. In vitro activity of APX001A against rare moulds using EUCAST and CLSI methodologies. J Antimicrob Chemother. 2019;74:1295-9.

53. Castanheira M, Duncanson FP, Diekema DJ, Guarro J, Jones RN, Pfaller MA. Activities of E1210 and comparator agents tested by CLSI and EUCAST broth microdilution methods against Fusarium and Scedosporium species identified using molecular methods. Antimicrob Agents Chemother. 2012;56:352-7.

54. Hager CL, Larkin EL, Long $L$, et al. In vitro and in vivo evaluation of the antifungal activity of APX001A/APX001 against Candida auris. Antimicrob Agents Chemother. 2018;62:e02319-7.

55. Jørgensen KM, Astvad KMT, Arendrup MC. In vitro activity of manogepix (APX001A) and comparators against contemporary molds: MEC comparison and preliminary experience with colorimetric MIC determination. Antimicrob Agents Chemother. 2020;64(8):e00730-20. https://doi.org/10.1128/AAC.00730-20.

56. Arendrup MC, Jørgensen KM. Manogepix (APX001A) displays potent in vitro activity against human pathogenic yeast, but with an unexpected correlation to fluconazole MICs. Antimicrob Agents Chemother. 2020;64(7):e00429-0. https://doi.org/10. 1128/AAC.00429-20.

57. Arendrup M, Chowdhary A, Jørgensen K, Meletiadis J. Manogepix (APX001A) in vitro activity against Candida auris. A head to head comparison of EUCAST and CLSI MICs. Antimicrob Agents Chemother. 2020. https://doi.org/10.1128/ AAC.00656-20 Fosmanogepix potently inhibits $\boldsymbol{C}$. auris.

58. Wiederhold NP, Najvar LK, Shaw KJ, Jaramillo R, Patterson HP, Olivo M, et al. In vivo efficacy of delayed therapy with the novel inositol acyltransferase inhibitor fosmanogepix (APX001) in a murine model of Candida auris invasive candidiasis. Open Forum Infect Dis. 2019;6:S60-1. https://doi.org/10.1093/ofid/ ofz359.131 In vivo efficacy against Candida auris.

59. Gebremariam T, Alkhazraji S, Alqarihi A, Wiederhold NP, Shaw KJ, Patterson TF, et al. Fosmanogepix (APX001) is effective in the treatment of pulmonary murine mucormycosis due to Rhizopus arrhizus. Antimicrob Agents Chemother. 2020;64(6): e00178-20. https://doi.org/10.1128/AAC.00178-20 In vivo data supporting fosmanogepix in treating mucormycosis.

60. Alkhazraji S, Gebremariam T, Alqarihi A, et al. Fosmanogepix (APX001) is effective in the treatment of immunocompromised mice infected with invasive pulmonary scedosporiosis or disseminated fusariosis. Antimicrob Agents Chemother. 2020;64(3): e01735-19. https://doi.org/10.1128/AAC.01735-19 In vivo model demonstrating successful activity against often panresistant mold species.

61. Kapoor M, Moloney M, Soltow QA, Pillar CM, Shaw KJ. Evaluation of resistance development to the Gwt1 inhibitor manogepix (APX001A) in Candida species. Antimicrob Agents Chemother. 2019;64:1. https://doi.org/10.1128/aac.01387-19.

62.• Amplyx.com. (2020) Amplyx announces positive top-line data in phase 2 clinical trial of novel antifungal fosmanogepix. amplyx. com/amplyx-announces-positive-top-line-data-in-phase-2clinical-trial-of-novel-antifungal-fosmanogepix/. Accessed 4 Aug 2020. Phase 2 clinical trial data recently released showing fosmanogepix's efficacy in treating invasive candidiasis caused by antifungal-resistant Candida species. All isolates were susceptible to the drug.

63. Oliver JD, Sibley GE, Beckmann N, et al. F901318 represents a novel class of antifungal drug that inhibits dihydroorotate dehydrogenase. Proc Natl Acad Sci USA. 2016;113:12809-14.

64. Wiederhold N. Review of the novel investigational antifungal olorofim. J Fungi. 2020;6:122. https://doi.org/10.3390/ jof6030122.

65. Rivero-Menendez O, Cuenca-Estrella M, Alastruey-Izquierdo A In vitro activity of olorofim (F901318) against clinical isolates of cryptic species of Aspergillus by EUCAST and CLSI methodologies. J Antimicrob Chemother. 2019;74:1586-90 In vitro activity against cryptic (often MDR) Aspergillus species.

66. Kirchhoff L, Dittmer S, Weisner A, Buer J, Rath P, Steinmann J. In vitro activity of olorofim (F901318) against fungi of the genus, Scedosporium and Rasamsonia as well as against Lomentospora prolificans, Exophiala dermatitidis and azole-resistant Aspergillus fumigatus. Int J Antimicrob. 2020:0924-8579. https://doi.org/10. 1016/j.ijantimicag.2020.106105 Olorofim has in vitro activity against MDR molds without reliable treatments available.

67. Wiederhold NP, Patterson HP, Birch M, Law D. Evaluation of the in vitro activity of olorofim against Fusarium species. In Proceedings of the 9th Trends in Medical Mycology, Nice, France, 11-19 October 2019.

68. Kirchhoff L, Dittmer S, Weisner A, Buer J, Rath P, and Steinmann J. Antibiofilm activity of antifungal drugs, including the novel drug olorofim, against Lomentospora prolificans. J Antimicrob Chemother 2020; https://doi.org/10.1093/jac/dkaa157. Olorofim has in vitro activity against Lomentospora spp. and penetrates biofilms. Currently antifungals have poor biofilm penetration. Biofilms significantly contribute to drug resistance.

69. Hope W, McEntee L, Livermore J, Whalley S, Johnson A, Farrington N, et al. Pharmacodynamics of the orotomides against Aspergillus fumigatus: new opportunities for treatment of multidrug-resistant fungal disease. mBio. 2017;8:e1157-17.

70. Negri C, Johnson A, McEntee L, Box H, Whalley S, Schwartz J, et al. Pharmacodynamics of the novel antifungal agent F901318 for acute sinopulmonary aspergillosis caused by Aspergillus flavus. J Infect Dis. 2017;217:1118-27.

71. Seyedmousavi S, Chang YC, Law D, Birch M, Rex JH, KwonChung KJ. Efficacy of olorofim (F901318) against Aspergillus fumigatus, A. nidulans, and A. tanneri in murine models of profound neutropenia and chronic granulomatous disease. Antimicrob Agents Chemother. 2019;63.

72. Fothergill AW, Wiederhold NP, Sibley G, Kennedy A, Oliver J, Birch M, et al. Spectrum of activity of F901318, the first agent 
from the newly discovered orotomide class of antifungals. ICAAC/ICC. 2015.

73. Wiederhold N, Najvar L, Jaramillo R, Olivo M, Birch M, Law D, et al. The orotomide olorofim is efficacious in an experimental model of central nervous system coccidioidomycosis. Antimicrob Agents Chemother. 2018;62:9. https://doi.org/10. 1128/AAC.00999-18.

74. Aigner M, Lass-Flörl C. Encochelated amphotericin B: is the oral availability of amphotericin B finally reached? J Fungi. 2020;6(2): 66. https://doi.org/10.3390/jof6020066.

75. Delmas G, Park S, Chen ZW, Tan F, Kashiwazaki R, Zarif L, et al. Efficacy of orally delivered cochleates containing amphotericin B in a murine model of aspergillosis. Antimicrob Agents Chemother. 2002;46:2704-7.

76. Mannino R, Perlin D. Oral dosing of encochleated amphotericin B $(\mathrm{CAmB})$ : rapid drug targeting to infected tissues in mice with invasive candidiasis. Scientific Presentations \& Publications Matinas Biopharma 2015. Available online: https://www. matinasbiopharma.com/media/scientific-presentationspublications. Accessed 12 Aug 2020.

77. Lu R, Hollingsworth C, Qiu J, Wang A, Hughes E, Xin X, et al. Efficacy of oral encochleated amphotericin B in a mouse model of cryptococcal meningoencephalitis. mBio. 2019;10:3. https://doi. org/10.1128/mBio.00724-19.

78. Skipper C, Atukunda M, Stadelman A, Engen N, Bangdiwala A, Hullsiek $\mathrm{KH}$, et al. Safety and tolerability of a novel oral formulation of amphotericin B: phase I EnACT trial. Antimicrob Agents Chemother. 2020. https://doi.org/10.1128/AAC.00838-20 Recent phase I data showing oral encochleated amphotericin B is easily tolerated with better safety compared to parenteral amphotericin $B$.

79. Kibathi L, Kumar P, Lionakis M, Urban A, Ferre E, McManus M, et al. 1413. A phase IIa efficacy, safety, tolerability and pharmacokinetic (PK) study of encochleated amphotericin B in patients with mucocutaneous (esophageal, oropharyngeal, vulvovaginal) candidiasis who are refractory or intolerant to standard nonintravenous therapies. Open Forum Infect Dis. 2018;5(1):S4355. https://doi.org/10.1093/ofid/ofy210.1244.

80. U.S. National Library of Medicine. ClinivalTrials.gov. NCT02971007. Safety and efficacy of oral encochleated amphotericin B (CAMB/MAT2203) in the treatment of vulvovaginal candidiasis (VVC). Available online: https:// clinicaltrials.gov/ct2/show/nct02971007. Accessed 12 Aug 2020.

81. Colley T, Alanio A, Kelly SL, Sehra G, Kizawa Y, Warrilow AGS, et al. In vitro and in vivo antifungal profile of a novel and long-acting inhaled azole, PC945, on aspergillus fumigatus infection. Antimicrob Agents Chemother. 2017;61(5):e02280-16. https://doi.org/10.1128/aac.02280-16.

82. Rudramurth $\mathrm{S}$, et al. In vitro antifungal activity of a novel topical triazole PC945 against emerging yeast Candida auris. J Antimicrob Chemother. 2019;74(10):2943-9. https://doi.org/10. 1093/jac/dkz280.

83. Colley T, Sehra G, Daly L, et al. Antifungal synergy of a topical triazole, PC945, with a systemic triazole against respiratory Aspergillus fumigatus infection. Sci Rep. 2019;9:9482. https:/ doi.org/10.1038/s41598-019-45890-w.

84. Pulmocide.com (2020). PC945 is a novel, potent, fungicidal antiAspergillus compound. Available at: http://pulmocide.com/ product/pc945/. Accessed on Aug 192020.

85. Yamashita K, Miyazaki T, Fukuda Y, et al. The novel arylamidine T-2307 selectively disrupts yeast mitochondrial function by inhibiting respiratory chain complexes. Antimicrob Agents Chemother. 2019;63:e00374-19.

86. Mitsuyama J, Nomura N, Hashimoto K, Yamada E, Nishikawa H, Kaeriyama M, et al. In vitro and in vivo antifungal activities of
T-2307, a novel arylamidine. Antimicrob Agents Chemother. 2008;52:1318-24.

87. Nishikawa H, Fukuda Y, Mitsuyama J, Tashiro M, Tanaka A, Takazono $\mathrm{T}$, et al. In vitro and in vivo antifungal activities of T-2307, a novel arylamidine, against Cryptococcus gattii: an emerging fungal pathogen. J Antimicrob Chemother. 2017;72: 1709-13.

88. Wiederhold NP, Najvar LK, Fothergill AW, Bocanegra R, Olivo M, McCarthy DI, et al. The novel arylamidine T-2307 demonstrates in vitro and in vivo activity against echinocandin-resistant Candida glabrata. J Antimicrob Chemother. 2016;71:692-5.

89. Wiederhold NP, Najvar LK, Fothergill AW, et al. The novel arylamidine T-2307 demonstrates in vitro and in vivo activity against Candida auris. J Antimicrob Chemother. 2019;64(3): e02198-19. https://doi.org/10.1128/AAC.02198-19.

90. Abe M, Nakamura S, Kinjo Y. Efficacy of T-2307, a novel arylamidine, against ocular complications of disseminated candidiasis in mice. J Antimicrob Chemother. 2019;74:1327-32.

91. Appilitherapeutics.com (2020). A novel, broad-spectrum, clinical stage antifungal to address severe and difficult-to-treat invasive fungal infections. Available online: https://www. appilitherapeutics.com/ati-2307. Accessed 10 Aug 2020. This agent was previously T-2307 until it lost funding for further development. Now Appili Therapeutics has recently acquired it to continue development.

92. Lockhart SR, Fothergill AW, Iqbal N, Bolden CB, Grossman NT, Garvey EP, et al. The investigational fungal Cyp51 inhibitor VT1129 demonstrates potent in vitro activity against Cryptococcus neoformans and Cryptococcus gattii. Antimicrob Agents Chemother. 2016;60:2528-31.

93. Nielsen K, Vedula P, Smith KD, Meya DB, Garvey EP, Hoekstra WJ, et al. Activity of VT-1129 against Cryptococcus neoformans clinical isolates with high fluconazole MICs. Med Mycol J. 2017;55(4):453-6. https://doi.org/10.1093/mmy/myw089.

94. Wiederhold NP, Najvar LK, Garvey EP, Brand SR, Xu X, Ottinger EA, et al. The fungal Cyp51 inhibitor VT-1129 is efficacious in an experimental model of cryptococcal meningitis. Antimicrob Agents Chemother. 2018;62.

95. Desai JV, et al. VT-1129 and VT-1161 have in vitro activity against Candida isolates from patients with chronic mucocutaneous candidiasis. Open Forum Infect Dis. 2017;3(1):1635. https:// doi.org/10.1093/ofid/ofw172.1335.

96. Wiederhold NP, Patterson HP, Tran BH, Yates CM, Schotzinger RJ, Garvey EP. Fungal-specific Cyp51 inhibitor VT-1598 demonstrates in vitro activity against Candida and Cryptococcus species, endemic fungi, including Coccidioides species, Aspergillus species and Rhizopus arrhizus. J Antimicrob Chemother. 2018;73(2):404-8. https://doi.org/10.1093/jac/dkx410.

97. Nishimoto A, Whaley S, Wiederhold N, Zhang Q, Yates C, Hoekstra W, et al. Impact of the major Candida glabrata triazole resistance determinants on the activity of the novel investigational tetrazoles VT-1598 and VT-1161. Antimicrob Agents Chemother. 2019;63.

98. Wiederhold NP, Lockhart SR, Najvar LK, et al. The fungal Cyp51-specific inhibitor VT-1598 demonstrates in vitro and in vivo activity against Candida auris. Antimicrob Agents Chemother. 2019;63:e02233-18.

99. Garvey EP, Sharp AD, Warn PA, Yates CM, Schotzinger RJ. The novel fungal CYP51 inhibitor VT-1598 is efficacious alone and in combination with liposomal amphotericin B in a murine model of cryptococcal meningitis. J Antimicrob Chemother. 2018;73(10): 2815-22. https://doi.org/10.1093/jac/dky242.

100. Wiederhold NP, Shubitz LF, Najvar LK, et al. The novel fungal Cyp51 inhibitor VT-1598 is efficacious in experimental models of central nervous system coccidioidomycosis caused by 
Coccidioides posadasii and Coccidioides immitis. Antimicrob Agents Chemother. 2018;62:e02258-17.

101. Hector RF, Zimmer BL, Pappagianis D. Evaluation of nikkomycins $\mathrm{X}$ and $\mathrm{Z}$ in murine models of coccidioidomycosis, histoplasmosis, and blastomycosis. Antimicrob Agents Chemother. 1990;34:587-93.

102. Shubitz LF, Roy ME, Nix DE, Galgiani JN. Efficacy of nikkomycin $Z$ for respiratory coccidioidomycosis in naturally infected dogs. Med Mycol. 2013;51:747-54.

103. Cheung Y-Y, Hui M. Effects of echinocandins in combination with nikkomycin $\mathrm{Z}$ against invasive Candida albicans bloodstream isolates and the fks mutants. Antimicrob Agents Chemother. 2017;61:e00619-7.

104. Nix DE, Swezey RR, Hector R, Galgiani JN. Pharmacokinetics of nikkomycin $\mathrm{Z}$ after single rising oral doses. Antimicrob Agents Chemother. 2009;53:2517-21.
105. Pfaller MA, Messer SA, Georgopapadakou N, Martell LA, Besterman JM, Diekema DJ. Activity of MGCD290, a Hos2 histone deacetylase inhibitor, in combination with azole antifungals against opportunistic fungal pathogens. J Clin Microbiol. 2009;47:3797-804.

106. Pfaller MA, Rhomberg PR, Messer SA, Castanheira M. In vitro activity of a Hos2 deacetylase inhibitor, MGCD290, in combination with echinocandins against echinocandin-resistant Candida species. Diagn Microbiol Infect Dis. 2015;81(4):259-63. https:// doi.org/10.1016/j.diagmicrobio.2014.11.008.

Publisher's Note Springer Nature remains neutral with regard to jurisdictional claims in published maps and institutional affiliations. 